\title{
Restoration Methods Selection for Wood Components of Chinese Ancient Architectures Based on TODIM with Single-Valued Neutrosophic Sets
}

\author{
Xiaolu. Long, ${ }^{1}$ Lizhi. Liu, ${ }^{2}$ Can. Xiao $\mathbb{i D}^{1}{ }^{1}$ Pengfei. Cheng $\left(\mathbb{D},{ }^{3,4}\right.$ and Chengxun. Fu ${ }^{4,5}$ \\ ${ }^{1}$ College of Architecture, Hunan University, Changsha 410082, China \\ ${ }^{2}$ Management Committee of Xiangtan Economic and Technological Development Zone, Xiangtan 411201, China \\ ${ }^{3}$ School of Business, Hunan University of Science and Technology, Xiangtan 411201, China \\ ${ }^{4}$ Hunan Engineering Research Center for Intelligent Decision Making and Big Data on Industrial Development, \\ Xiangtan 411201, China \\ ${ }^{5}$ Hunan Valin Xiangtan Iron and Steel Co. Ltd., Xiangtan 411101, China
}

Correspondence should be addressed to Can. Xiao; xiaocan@hnu.edu.cn and Pengfei. Cheng; 1180033@hnust.edu.cn

Received 19 March 2020; Accepted 27 April 2020; Published 13 May 2020

Guest Editor: Zaoli Yang

Copyright (c) 2020 Xiaolu. Long et al. This is an open access article distributed under the Creative Commons Attribution License, which permits unrestricted use, distribution, and reproduction in any medium, provided the original work is properly cited.

The selection of restoration methods for ancient architectures is of great significance for the protection of human cultural heritage. This paper proposes a novel restoration methods selection approach for wood components of Chinese ancient architectures, in which a multicriteria group decision-making (MCGDM) method with decision-making information is in the form of singlevalued neutrosophic sets (SNNSs). Firstly, it establishes an index system by comprehensively considering subjective and objective criteria. In addition, the best-worst method (BWM) and the entropy weight method are combined to produce index weights. Furthermore, the TODIM method is utilized by the single-valued neutrosophic sets to prioritize restoration methods. Finally, a specific case of wood component restoration is conducted to demonstrate the practicability of the proposed model. The robustness and effectiveness of the proposed method is verified by sensitivity analysis and comparison analysis.

\section{Introduction}

There are a large number of ancient architectural heritages with high historical, scientific, and artistic values in China. However, many of them are suffering not only from natural damage such as storms, fires, or insects but also from societal damage by rapid urbanization or inappropriate protective measures. Ancient architectures are nonrenewable, so it is important to choose appropriate restoration measures to reduce damage. The restoration for Chinese ancient architectures is a rather complex task. As the selection of restoration method is usually determined by a group of experts, it is a group decision-making problem affected by several factors such as situation of components, restoration process, restoration outcomes, and situation of commissioned restoration company.
With the development in architectural heritage protection practices, the restoration technologies have achieved rich achievements which have provided more options for the restoration of wood components of Chinese ancient architectures [1]. Although some progress has been made, there are still some issues to be addressed. Firstly, the common principles of restoration for ancient architectures include authenticity, reversibility, and minimal intervention. At present, the restoration technology of ancient buildings continues to advance, but it has not been applied scientifically and reasonably. If the repair method is not adopted properly, it will not only fail to effectively restore the ancient buildings but also cause irreversible secondary damage. Secondly, the evaluation information is usually partially. There is a lack of research on the standardization of selection process of restoration methods. Thirdly, in practice, the 
restoration methods selection is often determined based on experts' own knowledge and experience, which might lead to information insufficiency. Relevant research rarely uses quantitative methods to assess the decision-making process of Chinese ancient architectures' restoration.

In summary, the motivation of this paper is to propose a novel model of restoration methods selection for wood components of Chinese ancient architectures, which should improve the scientificity and standardization of decisionmaking during restoration methods selection and be conducive to the protection of ancient architectures. The rest of the paper is organized as follows. In Section 2, previous studies are reviewed in brief. In Section 3, some basic concepts and definitions about neutrosophic sets are introduced. Subsequently, a restoration methods selection model for wood components of Chinese ancient architectures based on MCGDM is developed in Section 4. In Section 5 , a case study is operated concretely, sensitivity analysis and comparative analysis are illustrated to verify the proposed model. Finally, Section 6 summarizes the content of the article and provides some possible directions of future study.

\section{Literature Review}

At present, a lot of restoration technologies for wood components of ancient architectures have been developed. The progress of modern technologies is mainly reflected in the use of new technical methods and new materials. For instance, Zaboklicki and Gebski [2] generalized that polymer composite inserts (such as epoxy resin, glass, and carbon fiber) enhance the continuity of wooden beams and expand their carrying capacity. Orlando et al. [3] focused on how to repair the ends of wooden beams, which decay easily. The study of Koike [4] reviews the development of wood biomass-sourced epoxy resin systems in Japan over the years. Dourado et al. [5] and Casals et al. [6] studied repaired wood structure adopting epoxy composites. Khelifa and Celzard [7] and Baratta and Corbi [8] recommended applying carbon fibre-reinforced polymer (CFRP) or fibre-reinforced polymer (FRP) to increase the bending capacity of timber structure. In real case, different technologies have different degrees of intervention on ancient architectures, and inappropriate technological advances might lead to negative effects; therefore, the choice of restoration methods must take the nonrenewability of Chinese ancient architectures into account. Therefore, we need to develop a scientific and reasonable measure to choose the restoration method.

Recently, some related indices affecting the restoration of Chinese ancient architectures have been discussed. Existing maintenance theory believes that the best protection and maintenance method would be a combination of active and passive maintenance; however, a lack of skilled operators is an obstacle to protect ancient architectures [9]. Fregonese et al. [10] believed that monitoring the condition of ancient building structures would be helpful in finding suitable restoration schemes. Gao et al. [11] even proposed an ancient timber buildings structural health assessment method based on fuzzy-element theory improved by asymmetric proximity. Lourenço [12] mentioned the importance of architectural characteristics such as structure and materials for the restoration of ancient architectures and pointed out that the value and authenticity of the building should be taken into consideration. By establishing the economic evaluation index system considering the cost, effect, technical, and other index for the ancient architecture protection scheme, Wang et al. [13] tried to choose a more economical ancient architecture protection scheme. Based on existing studies discussed above, they only focused on objective criteria such as component's conditions and technical methods. There are no studies considering both subjective and objective factors simultaneously, and the situation of restoration company has not been considered in the evaluation; no comprehensive index system has been established for the restoration of ancient architectures.

Neutrosophy has been introduced by Smarandache [14], whose fundamental proposition is that each concept has not only a certain degree of truth but also a degree of falsity and indeterminacy that must be considered independently from each other [15]. The neutrosophic set is similar to human thinking, reflecting the uncertainty caused by incomplete knowledge, incorrect knowledge acquisition, or random guessing [16]. Zhang et al. [17, 18] considered that the neutrosophic set is an effective tool for evaluating vague information. In the process of decision-making on the restoration methods of wood components, due to the uncertainty of actual restoration cases, experts' evaluation information is usually vague, and experts' evaluation information often represents emotional preference, which makes it difficult to be stated in an accurate quantitative form. We would better use neutrosophic numbers to describe the results of assessments [19, 20].

Methods for determining weights usually include subjective weighting methods and objective ones. In order to rank unknown weights, Rezaei [21] proposed the best-worst method (BWM) for multicriteria decision-making problem to calculate subjective weights. Compared with other methods to determine subjective weights (such as AHP), the BWM method has advantages in lesser data comparison and more consistent comparisons. However, the single use of subjective weights cannot guarantee the objectivity of evaluation results, and it will increase the difficulty in decision-makers' analysis and the possibility of errors. Hence, we shall use the entropy weight method. According to the characteristics of entropy, the entropy value shows the randomness and disorder of an index, and then, we can determine the degree of discreteness of the index. Therefore, a combination of BWM method with entropy weight method to compute index weights is necessary, which is not only based on the inherent laws of the index data but also expert experience. Thus, the result of index weights is more reliable [22].

TODIM (an acronym in Portuguese for interactive and multicriteria decision-making model) is based on the prospect theory [23]. Prospect theory [24] is considered to be the most typical behavioral decision theory and has been widely used in multicriteria decision problems [25-27]. TODIM method describes the dominance of each alternative over others fully considered the psychological behavior of decision-makers under risk $[28,29]$. In order to depict the 
psychological behavior of decision-makers, it is possible to modify parameters to reflect the preferences of decisionmakers [30-32]. To prioritize restoration methods, we shall use the TODIM method.

From the discussion above, the purpose of this study is to design a restoration methods selection model based on TODIM with single-valued neutrosophic sets for wood components of Chinese ancient architectures. This model is designed to help experts select the most appropriate restoration method. The contributions of this research are summarized as follows: (1) developing an index system for the restoration of wood components of Chinese ancient architectures; (2) using single-valued neutrosophic weighted average operator to aggregate experts' evaluation results, which make them more comprehensive and reliable; (3) applying the BWM method and entropy weight method to determine the index weights in case of unknown criteria and index weights; (4) introducing the TODIM method to obtain the ranking orders of alternatives; and (5) demonstrating the process of the proposed model by presenting an empirical study of a particular case. Also, to verify the validity and reliability of the model, sensitivity analysis and comparison analysis are conducted.

\section{Preliminaries}

In this section, we introduce some concepts and definitions, which will be useful in developing the wood components restoration methods selection model.

Definition 1 (neutrosophic set) (see [33]). Let $X$ be a space of points (objects), with a generic element in $X$ denoted by $x$. Then, a neutrosophic set $A$ in $X$ is characterized by three membership functions, including a truth-membership function $T_{A}$, an indeterminacy-membership function $I_{A}$, and a falsity-membership function $F_{A}$ and is defined as $A=\left\{\left\langle x, T_{A}(x), I_{A}(x), F_{A}(x)\right\rangle \mid x \in X\right\}$, where $T_{A}(x), I_{A}(x)$, and $F_{A}(x)$ are real standard or nonstandard subsets of $] 0^{-}, 1^{+}\left[\right.$, that is, $\left.T_{A}(x): X \longrightarrow\right] 0^{-}, 1^{+}\left[, I_{A}(x): X \longrightarrow\right]$ $0^{-}, 1^{+}\left[\right.$, and $\left.F_{A}(x): X \longrightarrow\right] 0^{-}, 1^{+}\left[\right.$. The sum of $T_{A}(x), I_{A}(x)$, and $F_{A}(x)$ is unrestricted and $0^{-} \leq T_{A}(x)+I_{A}(x)+F_{A}(x) \leq 3^{+}$.

Definition 2 (single-valued neutrosophic set) (see [34]). Let $X$ be a space of points (objects), with a generic element in $X$ denoted by $x$. A single-valued neutrosophic set (SVNS) $A$ in $X$ is characterized by truth-membership function $T_{A}$, indeterminacy-membership function $I_{A}$, and falsity-membership function $F_{A}$ with $T_{A}, I_{A}, F_{A} \in[0,1]$ for all $x$ in $X$. The sum of three memberships of a SVNS $A$, for all $x \in X$, $0 \leq T_{A}(x)+I_{A}(x)+F_{A}(x) \leq 3$.

Definition 3 (see [35]). Let $A$ and $B$ be two single-valued neutrosophic numbers, and then the operations can be defined as follows:

(1) $\lambda A=<1-\left(1-T_{A}\right)^{\lambda},\left(I_{A}\right)^{\lambda},\left(F_{A}\right)^{\lambda}>, \lambda>0$

(2) $A^{\lambda}=<\left(T_{A}\right)^{\lambda}, 1-\left(1-I_{A}\right)^{\lambda}, 1-\left(1-F_{A}\right)^{\lambda}>, \lambda>0$

(3) $A+B=<T_{A}+T_{B}-T_{A} \bullet T_{B}, I_{A} \bullet I_{B}, F_{A} \bullet F_{B}>$

(4) $A-B=\left\langle T_{A}-T_{B}, I_{A}+I_{B}-I_{A}-I_{B}, F_{A}+F_{B}-F_{A}-F_{B}>\right.$

(5) $A^{C}=\left\langle F_{A}, 1-I_{A}, 1-T_{A}>\right.$

Definition 4 (see [36]). Let $A=\left\{\left(x_{1} \mid<T_{A}\left(x_{1}\right), I_{A}\left(x_{1}\right), F_{A}\left(x_{1}\right)\right.\right.$ $\left.>), \ldots,\left(x_{n} \mid<T_{A}\left(x_{n}\right), I_{A}\left(x_{n}\right), F_{A}\left(x_{n}\right)>\right)\right\}$ and $B=\left\{\left(x_{1} \mid<T_{B}\left(x_{1}\right)\right.\right.$, $\left.\left.I_{B}\left(x_{1}\right), F_{B}\left(x_{1}\right)>\right), \ldots,\left(x_{n} \mid<T_{B}\left(x_{n}\right), I_{B}\left(x_{n}\right), F_{B}\left(x_{n}\right)>\right)\right\}$ be two SVNSs for $x_{i} \in X(i=1,2, \ldots, n)$. Then, the normalized Euclidean distance between $A$ and $B$ can be defined as follows:

$$
D(A, B)=\sqrt{\frac{1}{3 n} \sum_{i=1}^{n}\left\{\left(T_{A}\left(x_{i}\right)-T_{B}\left(x_{i}\right)\right)^{2}+\left(I_{A}\left(x_{i}\right)-I_{B}\left(x_{i}\right)\right)^{2}+\left(F_{A}\left(x_{i}\right)-F_{B}\left(x_{i}\right)\right)^{2}\right\}} .
$$

Definition 5 (see [36]). According to Majumdar et al.'s study, for single-valued neutrosophic set $A=\left\{\left\langle x, T_{A}(x)\right.\right.$, $\left.\left.I_{A}(x), F_{A}(x)\right\rangle \mid x \in X\right\}$, an entropy on neutrosophic set $A$ is computed as follows:

$$
E(A)=1-\frac{1}{n} \sum_{x_{i}}\left(T_{A}\left(x_{i}\right)+F_{A}\left(x_{i}\right)\right) \otimes\left|I_{A}\left(x_{i}\right)-I_{A^{C}}\left(x_{i}\right)\right| .
$$

Definition 6. (see [37]). The entropy weight of a neutrosophic set in a study by Tan et al. is shown as follows:

$$
W_{j}=\frac{\left(1-E\left(x_{j}\right)\right)}{\sum_{j}^{n}\left(1-E\left(x_{j}\right)\right)} .
$$

Definition 7. In the literature of Biswas et al. [38], fuzzification of SVNS $N(\sim)=\{(x \mid<T N(\sim)(x), I N(\sim)(x)$, and $F N(\sim)(x)>\mid x \in X\}$ is defined as follows:

$$
\mu_{\tilde{F}}(x)=1-\sqrt{\frac{1}{3}\left\{\left(1-T_{\widetilde{N}}(x)\right)^{2}+I_{\tilde{N}}(x)^{2}+F_{\widetilde{N}}(x)^{2}\right\}} .
$$

Definition 8 (see [39]). The single-valued neutrosophic weighted averaging (SVNWA) aggregation operator proposed by Ye's research can be shown as follows:

$$
\begin{aligned}
F_{A_{i}} & =\psi_{1} A_{1} \otimes \psi_{2} A_{2} \otimes \cdots \otimes \psi_{n} A_{n} \\
& =\left\langle\left\langle 1-\prod_{i=1}^{n}\left(1-T_{A_{i}}\right)^{\psi_{i}}, \prod_{k=1}^{n}\left(I_{A_{i}}\right)^{\psi_{i}}, \prod_{k=1}^{n}\left(F_{A_{i}}\right)^{\psi_{i}}\right\rangle,\right.
\end{aligned}
$$


where $\Psi=\left(\Psi_{1}, \Psi_{2}, \ldots, \Psi_{n}\right)$ is the weight vector of $A_{i}(i=1,2$, $\ldots, n), \Psi_{i} \in[0,1]$, and $\sum_{i=1}^{n} \psi_{i}=1$.

\section{The Proposed Selection Model for Wood Components Restoration Methods}

The selection of restoration methods for wood components of Chinese ancient architectures stands for a group decisionmaking problem seeking to find the best option. In actual cases, many indices could not be evaluated with accurate values in the decision-making process. Thus, the proposed model adopts single-valued neutrosophic sets to describe the characteristics of each index. The general process of the proposed model is shown in Figure 1. Details of the model will be explained in the rest of this section.

4.1. The Establishment of Index System. The restoration methods selection for wood components is generally decided by a group of $k$ experts. Based on analysis proposed in literature and experts' opinion, the evaluation of restoration for wood components of Chinese ancient architectures can be mainly measured by four categories, denoted by four criteria $A_{i}(i=1,2, \ldots, 4)$ : basic situation of components $\left(A_{1}\right)$, restoration process $\left(A_{2}\right)$, restoration outcomes $\left(A_{3}\right)$, and situation of commissioned restoration company $\left(A_{4}\right)$. Moreover, under each criterion, there are several subcriteria, which influence the selection of restoration methods. Hence, an index system is established, as shown in Table 1.

4.2. The Acquisition of Evaluation Matrix. The aspects to be considered in evaluating wood components restoration of Chinese ancient architectures include practical cases, experts, wood components to be repaired, and diverse evaluation indices. Different experts may make different assessments based on their education background, distinct experiences, different assessment criteria, and different restoration methods suitable for different wood component's conditions. Because of the ambiguity and complexity of information, the evaluation values of indices often cannot be expressed in clear numbers. Decision-makers are more inclined to use fuzzy numbers such as linguistic terms with multiple granularities to evaluate indices. Thus, in this section, the evaluation results obtained from experts can be transformed into single-valued neutrosophic numbers. Hence, we can get the evaluation matrix $R=\left(r_{i j}\right)$. The specific process is as follows:

Step 1. Obtaining linguistic terms with
multigranularity.

In practical cases, experts may choose linguistic terms based on their preferences. Therefore, due to semantic differences in linguistic terms, different experts may have different evaluation values. Evaluation values of indices are given in the form of multigranularity linguistic terms [40] by an expert group consisting of different experts. Therefore, a linguistic term set containing ordered linguistic terms needs to be set in advance. Different linguistic term sets show different characteristics of membership function. For example, linguistic term set $\left\{m_{0}, m_{1}, m_{2}, m_{3}, m_{4}, m_{5}, m_{6}, m_{7}, m_{8}\right\}$ can be donated in linguistic terms as \{extremely bad, very bad, bad, medium bad, medium, medium good, good, very good, extremely good $\}$, while linguistic term set $\left\{l_{0}, l_{1}, l_{2}, l_{3}, l_{4}, l_{5}, l_{6}\right\}$ can be donated in linguistic terms as\{very bad, bad, medium bad, medium, medium good, good, very good , but different experts may have different understanding of these terms [41]. Hence, it would be better that experts provide evaluation values for different restoration methods according to a preset linguistic term set.

Step 2. Transforming the evaluation information from experts' questionnaire according to the symmetric linguistic evaluation scale into single-valued neutrosophic numbers with truth, indeterminacy, and falsity.

The linguistic term set in this study is \{extremely high, very high, high, medium high, medium, medium low, low, very low, extremely low\}, including nine granular linguistic terms. The linguistic terms along with SVNNs are defined in Table 2 as given in the literature [38] to rate each alternative with respect to each index.

Step 3. Determining the weights of experts.

In accordance with the different backgrounds and experiences of different experts, the importance of decision-making experts differs. In this paper, experts' decision power can be expressed in the linguistic terms set \{very important, important, medium, unimportant, very unimportant\}, and the corresponding single-valued neutrosophic numbers are shown in Table 3 . The weight of expert is donated as $e_{k}(k=1,2, \ldots, n)$; according to equation (4), the weights of experts can be computed by the following equation [38]:

$$
e_{k}=\frac{u_{k}}{\sum_{k=1}^{l} u_{k}}=\frac{1-\sqrt{\left\{\left(1-T^{k}\right)^{2}+\left(I^{k}\right)^{2}+\left(F^{k}\right)^{2}\right\} / 3}}{\sum_{k=1}^{l}\left(1-\sqrt{\left\{\left(1-T^{k}\right)^{2}+\left(I^{k}\right)^{2}+\left(F^{k}\right)^{2}\right\} / 3}\right)} .
$$

Step 4. Aggregating the neutrosophic numbers into $R=\left(r_{i j}\right)$.

We use the single-valued neutrosophic weighted averaging (SVNWA) aggregation operator described by equation (5) introduced in Section 2 to aggregate the neutrosophic numbers. Then, we shall get the evaluation matrix $R=\left(r_{i j}\right)$.

4.3. The Assignment of Index Weights. In this section, we employ a combination of subjective and objective weights to determine the index weights. To begin with, we utilize an efficient method called BWM to calculate subjective weights of four criteria. Then, we adopt the principle of objectivity to calculate the entropy weight of each index. Based on the subjective-objective method to determine the synthetic weight of each index, the detailed procedures are as follows. 


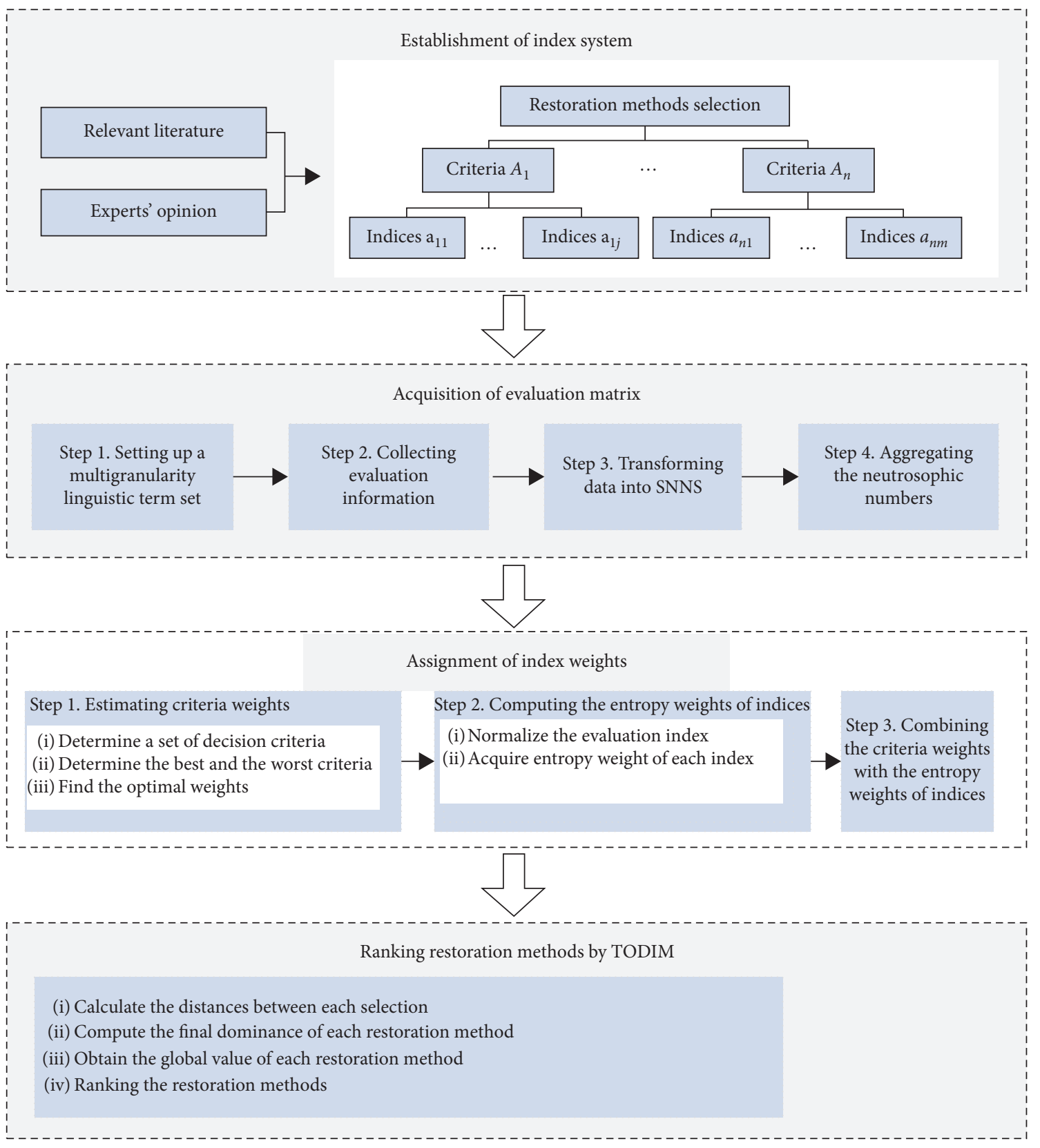

FIGURE 1: Summary of the process of the proposed model.

4.3.1. Estimating Criteria Weights. BWM method, introduced by Raize, is the newest method to solve multicriteria decision-making problems [42]. In this section, we apply it to calculate the weights of four criteria [43]:

Step 1. Determining a set of decision criteria. We should consider the criteria $\left\{A_{1}, A_{2}, \ldots, A_{n}\right\}$ that should be used to make a decision.

Step 2. The best (e.g., most desirable and most important) and the worst (e.g., least desirable and least important) criteria are determined by decision-maker in general.

Step 3. In the light of the BWM questionnaire, the preference of the best criterion over all the other criteria using a number between 1 and 9 should be determined. The resulting best-to-others vector would be $A_{B}=\left(a_{B 1}\right.$, $\left.a_{B 2}, \ldots, a_{B n}\right)$, where $a_{B j}$ indicates the preference of the best criterion $B$ over criterion $j$. It is clear that $a_{B B}=1$.

Step 4. According to the BWM questionnaire, the preference of all the criteria over the worst criterion, using number between 1 and 9, should be determined. The resulting others-to-worst vector would be $A_{W}=\left(a_{1 W}, a_{2 W}, \ldots, a_{n W}\right)$, where $a_{j W}$ indicates the preference of the criterion $j$ over the worst criterion $W$. It is clear that $a_{W W}=1$.

Step 5. Finding the optimal weights $\left\{w_{1}^{*}, w_{2}^{*}, \ldots, w_{n}^{*}\right\}$ by solving (7). The optimal weight for the criterion is the one where for each pair of $w_{B} / w_{j}$ and $w_{j} / w_{W}$, we 
TABLE 1: Index system of restoration methods selection for wood components of Chinese ancient architectures.

\begin{tabular}{|c|c|c|c|}
\hline Criteria & Index & Definition & $\begin{array}{l}\text { Index } \\
\text { type }\end{array}$ \\
\hline \multirow{6}{*}{$\begin{array}{l}\text { Basic situation of } \\
\text { components }\left(A_{1}\right)\end{array}$} & Used years $\left(a_{11}\right)$ & $\begin{array}{l}\text { The degree that the used years of the component is suitable } \\
\text { for these restoration methods }\end{array}$ & Benefit \\
\hline & Material properties $\left(a_{12}\right)$ & $\begin{array}{l}\text { The degree that the material properties of the component is } \\
\text { suitable for these restoration methods }\end{array}$ & Benefit \\
\hline & Structural rationality $\left(a_{13}\right)$ & $\begin{array}{l}\text { The degree of rationality that the structure of the component } \\
\text { is suitable for these restoration methods }\end{array}$ & Benefit \\
\hline & Cause of damage $\left(a_{14}\right)$ & $\begin{array}{l}\text { The cause of damage of the component is suitable for these } \\
\text { restoration methods }\end{array}$ & Benefit \\
\hline & Severity of damage $\left(a_{15}\right)$ & $\begin{array}{l}\text { The degree of severity that the damage of the component is } \\
\text { suitable for these restoration methods }\end{array}$ & Benefit \\
\hline & $\begin{array}{l}\text { Impact on neighboring } \\
\text { components }\left(a_{16}\right)\end{array}$ & $\begin{array}{l}\text { The degree of impact on neighboring components using } \\
\text { these restoration methods }\end{array}$ & Cost \\
\hline \multirow{4}{*}{ Restoration process $\left(A_{2}\right)$} & Restoration expense $\left(a_{21}\right)$ & The cost spent for the restoration & Cost \\
\hline & Material acquisition $\left(a_{22}\right)$ & $\begin{array}{c}\text { The degree of difficulty in obtaining materials for the } \\
\text { restoration }\end{array}$ & Cost \\
\hline & Process complexity $\left(a_{23}\right)$ & The degree of process complexity in the restoration & Cost \\
\hline & Requirements for equipment $\left(a_{24}\right)$ & The level of requirements for equipment in the restoration & Cost \\
\hline \multirow{6}{*}{ Restoration outcomes $\left(A_{3}\right)$} & $\begin{array}{l}\text { Probability of authenticity } \\
\text { restoration }\left(a_{31}\right)\end{array}$ & $\begin{array}{l}\text { The probability of fixing the components without changing } \\
\text { the original materials and processing methods of the } \\
\text { component }\end{array}$ & Benefit \\
\hline & $\begin{array}{l}\text { Degree of preservation of } \\
\text { historical information }\left(a_{32}\right)\end{array}$ & $\begin{array}{l}\text { The probability that historical information shall not be } \\
\text { destroyed during restoration }\end{array}$ & Benefit \\
\hline & $\begin{array}{l}\text { Probability of irreversible effects } \\
\text { on the original component }\left(a_{33}\right)\end{array}$ & $\begin{array}{l}\text { The probability that the original component could not be } \\
\text { returned to the prerepair state after restoration if an error } \\
\text { occurred during the repair process }\end{array}$ & Cost \\
\hline & $\begin{array}{l}\text { Possibility of reversible effects on } \\
\text { the original component }\left(a_{34}\right)\end{array}$ & $\begin{array}{l}\text { The possibility that the original component could be } \\
\text { returned to the prerepair state after restoration if an error } \\
\text { occurred during the repair process }\end{array}$ & Benefit \\
\hline & Service life after restoration $\left(a_{35}\right)$ & $\begin{array}{l}\text { The length of time that the component may be used after } \\
\text { restoration }\end{array}$ & Benefit \\
\hline & Difficulty of maintenance $\left(a_{36}\right)$ & $\begin{array}{l}\text { The difficulty in maintenance of the component after } \\
\text { restoration }\end{array}$ & Cost \\
\hline \multirow{2}{*}{$\begin{array}{l}\text { Situation of commissioned } \\
\text { restoration company }\left(A_{4}\right)\end{array}$} & $\begin{array}{l}\text { Teamwork capacity }\left(a_{41}\right) \\
\text { Proficiency }\left(a_{42}\right)\end{array}$ & $\begin{array}{l}\text { The cooperation efficiency of the restoration team } \\
\text { Restoration team professionals' degree of skills }\end{array}$ & $\begin{array}{l}\text { Benefit } \\
\text { Benefit }\end{array}$ \\
\hline & $\begin{array}{l}\text { Advanced restoration equipment } \\
\qquad\left(a_{43}\right)\end{array}$ & The performance of the restoration equipment & Benefit \\
\hline
\end{tabular}

TABLE 2: Linguistic terms for rating the alternatives with SVNNs.

\begin{tabular}{lc}
\hline Linguistic terms & \multicolumn{1}{c}{ SVNNs } \\
\hline Extremely good/high & $<1.00,0.00,0.00>$ \\
Very good/high & $<0.90,0.10,0.05>$ \\
Good/high & $<0.80,0.20,0.15>$ \\
Medium good/high & $<0.65,0.35,0.30>$ \\
Medium/fair & $<0.50,0.50,0.45>$ \\
Medium bad/medium low & $<0.35,0.65,0.60>$ \\
Bad/low & $<0.20,0.75,0.80>$ \\
Very bad/low & $<0.10,0.85,0.90>$ \\
Extremely bad/low & $<0.05,0.90,0.95>$ \\
\hline
\end{tabular}

TABLE 3: Linguistic terms for rating the importance of experts with SVNNs.

\begin{tabular}{lc}
\hline Linguistic terms & SVNNs \\
\hline Very important (VI) & $<0.90,0.10,0.05>$ \\
Important (I) & $<0.80,0.20,0.15>$ \\
Medium (M) & $<0.50,0.40,0.45>$ \\
Unimportant (UI) & $<0.35,0.60,0.70>$ \\
Very unimportant (VUI) & $<0.10,0.80,0.90>$ \\
\hline
\end{tabular}

have $w_{B} / w_{j}=a_{B j}$ and $w_{j} / w_{W}=a_{j W}$. In order to meet these conditions for all $j$, we should find a solving method where the maximum absolute differences $\left|\left(w_{B} / w_{j}\right)-a_{B j}\right|$ and $\left|\left(w_{j} / w_{w}\right)-a_{j w}\right|$ for all $j$ are minimized. Considering the nonnegativity and condition for the weights, the following problem results:

$$
\min \max _{j}\left\{\left|\frac{w_{B}}{w_{j}}-a_{B j}\right|,\left|\frac{w_{j}}{w_{w}}-a_{j w}\right|\right\}
$$

s.t.

$$
\begin{aligned}
& \sum_{j} w_{j}=1 \\
& w_{j} \geq 0, \quad \text { for all } j .
\end{aligned}
$$

Equation (7) is equivalent to the following equation: 


$$
\begin{aligned}
& \min \\
& \text { s.t. } \\
& \left|\frac{w_{B}}{w_{j}}-a_{B j}\right| \leq \xi, \quad \text { for all } j \\
& \left|\frac{w_{j}}{w_{w}}-a_{j w}\right| \leq \xi, \quad \text { for all } j \\
& \sum_{j} w_{j}=1 \\
& w_{j} \geq 0, \quad \text { for all } j .
\end{aligned}
$$

Solving equation (8), the optimal weights $\left(w_{1}^{*}, w_{2}^{*}, \ldots, w_{n}^{*}\right)$ and $\xi^{*}$ can be obtained.

Then, the consistency ratio using $\xi^{*}$ and the corresponding consistency index can be calculated using the following formula:

$$
\text { consistency ratio }=\frac{\xi^{*}}{\text { consistency index }} .
$$

The maximum values of $\xi$ (consistency index) for different values of $a_{B w}$ are shown in Table 4 .

Consistency ratio $\in[0,1]$ : the value approaching 0 proved more consistency; instead, the value close to 1 shows less consistency. If the consistency ratio is $\leq 0.1$, it indicates a very good consistency. If not, we should revise $a_{B j}$ and $a_{j W}$ to make the solution more consistent.

In the case when the number of criteria exceeds three, the BWM introduced above is limited in deriving the unique optimal weight vector. This leads to multiple optimal solutions. The improved method presented in the study of Rezaei [21] is used to acquire optimal weights with $n$ criteria. If we use $\left\{\left|\mathrm{w}_{B}-a_{B j} w_{j}\right|,\left|w_{j}-a_{j w} w_{w}\right|\right\}$ instead of $\left\{\left|\left(w_{B} / w_{j}\right)-a_{B j}\right|,\left|\left(w_{j} / w_{w}\right)-a_{j w}\right|\right\}$, the problem can be solved as follows:

$$
\begin{array}{ll}
\min _{\max } & \left\{\left|\mathrm{w}_{B}-a_{B j} w_{j}\right|,\left|w_{j}-a_{j w} w_{w}\right|\right\}, \\
\text { s.t. } & \sum_{j} w_{j}=1 \\
w_{j} \geq 0, \quad & \text { for all } j .
\end{array}
$$

Equation (10) can be transferred to a linear programming problem as follows:

$$
\begin{array}{ll}
\min & \xi^{L} \\
\text { s.t. } & \\
\left|w_{B}-a_{B j} w_{j}\right| \leq \xi^{L}, & \text { for all } j \\
\left|w_{j}-a_{j w} w_{w}\right| \leq \xi^{L}, & \text { for all } j \\
\sum_{j} w_{j}=1 & \\
w_{j} \geq 0, & \text { for all } j .
\end{array}
$$

Equation (11) is a linear program problem, which can compute the unique optimal weight $\left\{w_{1}^{*}, w_{2}^{*}, \ldots, w_{n}^{*}\right\}$.
Therefore, we can obtain the weight vector $\left\{w_{1}, w_{2}, w_{3}, w_{4}\right\}$ of basic situation of components $A_{1}$, restoration process $A_{2}$, restoration outcomes $A_{3}$, and situation of commissioned restoration company $A_{4}$.

4.3.2. Calculating Index Weights. In this section, we follow the combination of subjective and objective principles to compute the index weights. Firstly, we use entropy weight method to compute the entropy weight of each index. Then, we obtain synthesized weight of each index by combining criteria weights with entropy weights. The detailed processes are as follows:

Step 1. Normalizing the evaluation index.

The normalization equation [35] for neutrosophic numbers is as in the following equation. Then, we obtain the normalization matrix $R=\left(\beta_{i j}\right)$ :

$r_{i j}= \begin{cases}T_{i j}, I_{i j}, F_{i j}, & \text { if } j \text { is benefit index, } \\ 1-T_{i j}, 1-I_{i j}, 1-F_{i j}, & \text { if } j \text { is cost index. }\end{cases}$

Step 2. Computing the entropy weights of indices.

According to equations (2) and (3) introduced in Section 2, first, we shall compute the entropy value of each index $E\left(x_{j}\right)$. Then, we can acquire the entropy weight $w_{j}$ of each index based on the evaluation matrix. Step 3. Calculating combination weights.

It is important to confirm the weights of the evaluation indicators of the restoration methods, which affects the accuracy of the evaluation results. Subjective weight method relies on knowledge and experience of the experts, and the evaluation results are usually arbitrary. The objective weighting method is based on objective actual conditions and can reduce subjective arbitrariness. So, a combination of subjective and objective methods is necessary. The optimal weight vector of criteria is $w_{i}^{*}$ according to the explanation of BWM in Section 4.3.1. Then, we compute a comprehensive index weight $H_{i j}$ which combines the subjective weights of criteria with the entropy weights of indices according to the following equation [44]:

$$
H_{i j}=\frac{w_{i}^{*} \times w_{j}}{\sum_{i=1}^{n} w_{i}^{*} \times w_{j}} .
$$

4.4. The Process of Decision-Making Based on TODIM. To solve multicriteria decision-making problems, Gomes and Lima [45] proposed the TODIM method based on prospect theory. Lourenzutti and Krohling [46] introduced the primary principle of the TODIM method. Recently, an unexpected initiative of the TODIM method was pointed out by Lourenzutti et al., which states that both the losses and the gains should be amplified proportionally by the criterion weight, and then applied to prospect function. The suggested modification in the $\phi_{c}$ function is as follows: 
TABle 4: Consistency index (CI) table.

\begin{tabular}{lccccccccc}
\hline$a_{B w}$ & 1 & 2 & 3 & 4 & 5 & 6 & 7 & 8 & 9 \\
\hline Consistency index $(\max \xi)$ & 0.00 & 0.44 & 1.00 & 1.63 & 2.30 & 3.00 & 3.73 & 4.47 & 5.23 \\
\hline
\end{tabular}

$$
\phi_{c}\left(X_{i}, X_{j}\right)= \begin{cases}\sqrt{w_{c}\left(s_{i c}-s_{j c}\right)}, & \text { if } s_{i c} \geq s_{j c} \\ -\frac{1}{\theta} \sqrt{w_{c}\left(s_{j c}-s_{i c}\right)}, & \text { otherwise. }\end{cases}
$$

The selection of restoration methods for wood components of Chinese ancient architectures is a multicriteria group decision-making (MCGDM) problem consisting of expert $e_{k}(k=1,2, \ldots, n)$, denoted by $\omega=\left\{e_{1}, e_{2}, \ldots, e_{k}\right\}$. Suppose that there exist $n$ restoration methods $X_{i}(i=1,2,3, \ldots, n)$, denoted by $X=\left\{X_{1}, X_{2}, \ldots, X_{n}\right\}$. In this part, we use the TODIM method to solve the restoration methods selection problem. The main procedure is generalized as follows:

Step 1. Calculating the distances $d_{i j}$ between each selection based on equation (1).

Step 2. Computing the final dominance of restoration method $X_{i}$ over each restoration method $X_{k}$ under each criterion $A_{j}$ as follows [23]:

$$
\begin{gathered}
\delta\left(X_{i}, X_{k}\right)=\sum_{j=1}^{n} \phi_{j}\left(X_{i}, X_{k}\right), \quad \forall(i, k) \text { where } \\
\phi_{j}\left(X_{i}, X_{k}\right)= \begin{cases}\sqrt{w_{j} d\left(b_{i j}, b_{k j}\right)}, & \text { if } b_{i j}>b_{k j}, \\
0, & \text { if } b_{i j}=b_{k j}, \\
-\frac{1}{\theta} \sqrt{w_{j} d\left(b_{i j}, b_{k j}\right)}, & \text { otherwise, }\end{cases}
\end{gathered}
$$

where $d\left(b_{i j}, b_{k j}\right)$ is the distance between $b_{i j}$ and $b_{k j}$. The $w_{j}$ is the weight of the $j$ criterion calculated by the BWM method. In this paper, we use comprehensive index weight $H_{i j}$ instead of $w_{j}$, where $H_{i j}$ is the index weight calculated by a combination of the criteria weights calculated by BWM and the indices weights obtained by the entropy weight method. So, we shall apply equation (16) to acquire the final dominance of restoration method $X_{i}$ over each restoration method $X_{k}$ under each index $a_{i j}$ :

$$
\begin{gathered}
\delta\left(X_{i}, X_{k}\right)=\sum_{j=1}^{n} \phi_{j}\left(X_{i}, X_{k}\right), \quad \forall(i, k) \text { where } \\
\phi_{j}\left(X_{i}, X_{k}\right)= \begin{cases}\sqrt{H_{i j} d\left(b_{i j}, b_{k j}\right)}, & \text { if } b_{i j}>b_{k j}, \\
0, & \text { if } b_{i j}>b_{k j}, \\
-\frac{1}{\theta} \sqrt{H_{i j} d\left(b_{i j}, b_{k j}\right)}, & \text { otherwise. }\end{cases}
\end{gathered}
$$

Step 3. The global value of restoration method $X_{i}$ is obtained as follows [23]:

$$
\varepsilon_{i}=\frac{\sum_{j} \delta\left(X_{i}, X_{k}\right)-\min _{i} \sum_{j} \delta\left(X_{i}, X_{k}\right)}{\max _{i} \sum_{j} \delta\left(X_{i}, X_{k}\right)-\min _{i} \sum_{j} \delta\left(X_{i}, X_{k}\right)}
$$

Step 4. Ranking the restoration methods $X i$ by their value $\varepsilon_{i}$.

According to the value $\varepsilon_{i}$, we could select restoration method $X_{i}$. The higher the value $\varepsilon_{i}$, the better the restoration method $X_{i}$.

\section{Empirical Study}

The proposed model is applied to solve the selection problem of restoration methods for wood components of Chinese ancient architectures. A Chinese ancient architecture called "Fuhoutang" needed to be restored. Fuhoutang is a statelevel cultural legacy, which was constructed during the Xianfeng period of Qing Dynasty. The management authority of Fuhoutang commissioned company $R$ to restore it. One of the components to be restored is a beam at the bottom of the wooden frame in the hall, donated by $C_{1}$. The original information of $C_{1}$ is outlined in Table 5. Four experts, donated by $\mathrm{DM}_{1}, \mathrm{DM}_{2}, \mathrm{DM}_{3}$, and $\mathrm{DM}_{4}$, respectively, studied the condition of $C_{1}$ and then provided four possible restoration methods, including stabilizing by outer iron hoop, inserting internal steel, filling with epoxy, and replacing the component, which are denoted by $X_{1}, X_{2}, X_{3}$, and $X_{4}$ (shown in Figure 2). These four restoration methods are evaluated by experts through several evaluation indices to determine the advantages and disadvantages of each method, and the evaluation index system is listed in Table 1.

According to the selection model presented in Section 3, first, we get the evaluation matrix through the conditions of the wood component to be restored, restoration process, the possible outcomes, and restoration company's conditions. Then, the index weights are calculated by a combination of criteria weights and entropy weights. Finally, the ranking order of the four restoration methods can be acquired by using the TODIM method. Furthermore, the effectiveness and reliability of the proposed model are verified by sensitivity analysis and comparison analysis.

5.1. Evaluation Matrix. The evaluation matrix is obtained from experts according to the evaluation index system. In the light of the evaluation method proposed in Section 4.2, experts evaluate the four methods, and the index evaluation value is represented by linguistic terms with multigranularity. We transform the linguistic terms into singlevalued neutrosophic numbers (SVNNs) according to Table 2. Based on experts' distinct experience, the importance of four experts differs from each other, as shown in Table 6. According to Table 3, the importance of each expert could be 
TABLE 5: The information of the condition of the specific component to be repaired.

\begin{tabular}{lc}
\hline$C_{1}$ & Component's condition \\
\hline Used years & About 150 years \\
Original material & Chinese fir \\
The cause of & Crack, decay \\
damage & C
\end{tabular}

The beam is $35 \mathrm{~cm}$ in diameter and 4.2 meters long. The crack is about $80 \mathrm{~cm}$ long, about $4 \mathrm{~cm}$ wide, and about $5 \mathrm{~cm}$ Damage situation deep, which is caused by material properties. Near the crack, a decay of about $8 \mathrm{~cm}$ in width, $20 \mathrm{~cm}$ in length, and $3 \mathrm{~cm}$ in depth was eroded by bugs

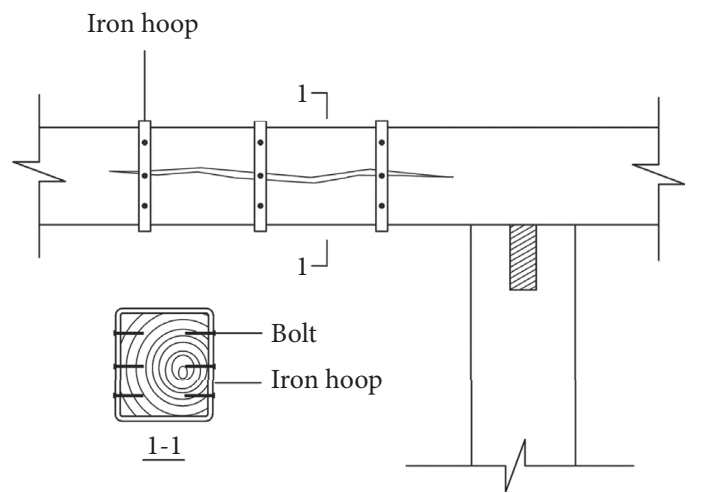

(a)

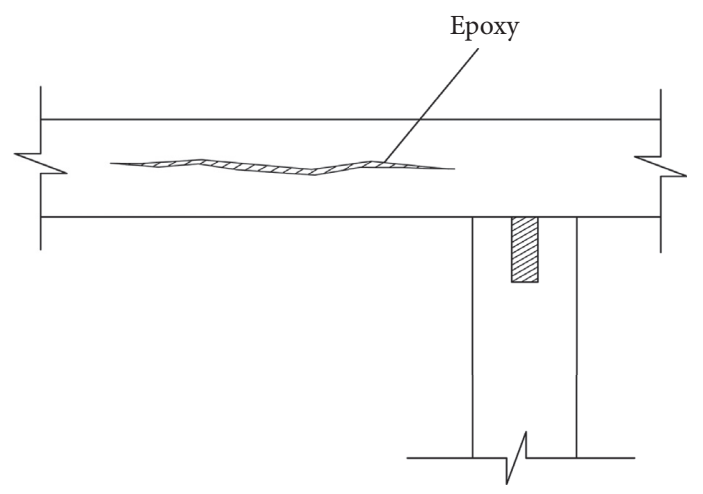

(c)

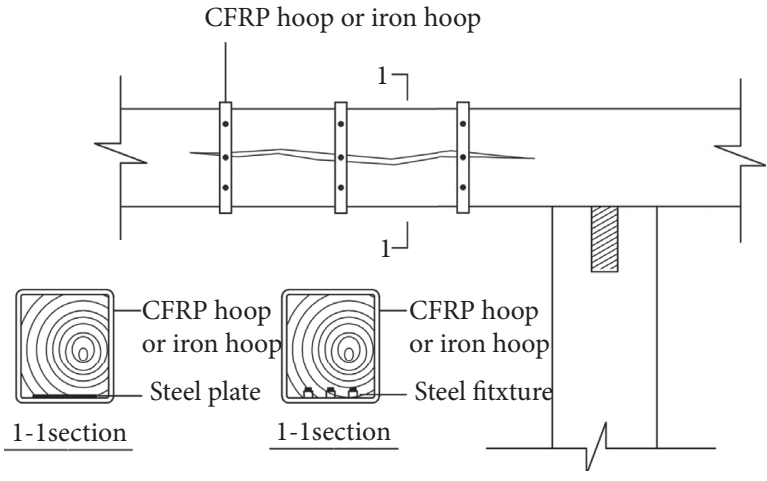

(b)

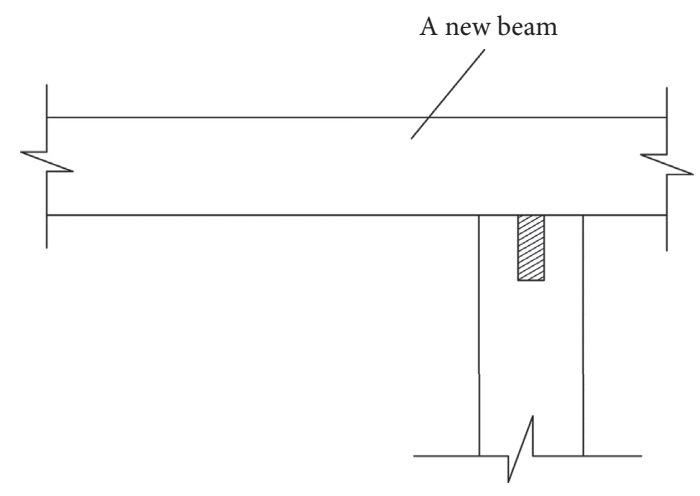

(d)

FIGURE 2: Four alternative restoration methods for $C_{1}$ : (a) stabilizing by outer iron hoop; (b) inserting internal steel (CFRP stands for carbon fibre-reinforced polymer); (c) filling with epoxy; (d) replacing the component.

TABLE 6: Importance of experts expressed with SVNNs.

\begin{tabular}{lcccc}
\hline & $\mathrm{DM}_{1}$ & $\mathrm{DM}_{2}$ & $\mathrm{DM}_{3}$ & $\mathrm{DM}_{4}$ \\
\hline Linguistic terms & $\mathrm{M}$ & $\mathrm{M}$ & $\mathrm{I}$ & $\mathrm{VI}$ \\
SVNNs & $<0.50,0.40,0.45>$ & $<0.50,0.40,0.45>$ & $<0.80,0.20,0.15>$ & $<0.90,0.10,0.05>$ \\
\hline
\end{tabular}

described by linguistic terms with its relevant SVNNs. Therefore, the weights of experts $\left(e_{k}\right)$ are determined by using equation (6): $e_{1}=0.1950, e_{2}=0.1950, e_{3}=0.2899$, and $e_{4}=0.3201$. Then, the evaluation values of these indices do need to be aggregated. We shall use equation (4), the singlevalued neutrosophic weighted averaging (SVNWA) aggregation operator to aggregate these indices. Finally, we obtain the evaluation matrix $R=\left(r_{i j}\right)$ based on single-valued neutrosophic sets outlined in Table 7.
5.2. The Calculation of Index Weights. According to the BWM method introduced in Section 4.3.1, we calculate the subjective weights of four criteria. Firstly, the criteria shown in Table 1 are denoted by $A_{1}$ to $A_{4}$; among them, basic situation of components $\left(A_{1}\right)$ is the most important criterion, and restoration process $\left(A_{2}\right)$ is the least important criterion, which is confirmed by experts. Secondly, the pairwise comparison vectors for the best and the worst criteria are outlined in Tables 8 and 9. Table 8 represents that 
TABLe 7: The aggregated SVNNs-based decision matrix.

\begin{tabular}{|c|c|c|c|c|c|}
\hline \multirow{2}{*}{ Criteria } & \multirow{2}{*}{ Index } & \multicolumn{4}{|c|}{ Alternatives } \\
\hline & & $X_{1}$ & $X_{2}$ & $X_{3}$ & $X_{4}$ \\
\hline \multirow{6}{*}{$A_{1}$} & $a_{11}$ & $<0.719,0.281,0.229>$ & $<0.5,0.5,0.45>$ & $<0.777,0.223,0.172>$ & $<0.465,0.525,0.491>$ \\
\hline & $a_{12}$ & $<0.761,0.239,0.187>$ & $<0.506,0.494,0.442>$ & $<0.8,0.2,0.15>$ & $<0.545,0.455,0.398>$ \\
\hline & $a_{13}$ & $<0.704,0.296,0.240>$ & $<0.648,0.352,0.298>$ & $<0.733,0.267,0.214>$ & $<0.262,0.709,0.715>$ \\
\hline & $a_{14}$ & $<0.625,0.375,0.325>$ & $<0.473,0.526,0.476>$ & $<0.777,0.223,0.172>$ & $<0.453,0.547,0.496>$ \\
\hline & $a_{15}$ & $<0.625,0.375,0.325>$ & $<0.579,0.421,0.370>$ & $<0.8,0.2,0.15>$ & $<0.159,0.791,0.841>$ \\
\hline & $a_{16}$ & $<0.191,0.776,0.790>$ & $<0.492,0.507,0.456\rangle$ & $<0.186,0.782,0.796>$ & $<1,0,0>$ \\
\hline \multirow{4}{*}{$A_{2}$} & $a_{21}$ & $<0.281,0.697,0.690>$ & $<0.765,0.235,0.183>$ & $<0.534,0.466,0.416>$ & $<0.714,0.286,0.232>$ \\
\hline & $a_{22}$ & $<0.181,0.769,0.819>$ & $<0.565,0.435,0.384>$ & $<0.413,0.587,0.536>$ & $<0.765,0.235,0.183>$ \\
\hline & $a_{23}$ & $<0.35,0.65,0.6>$ & $<0.825,0.175,0.121>$ & $<0.456,0.544,0.493>$ & $<0.766,0.234,0.169>$ \\
\hline & $a_{24}$ & $<0.305,0.680,0.658>$ & $<0.8,0.2,0.15>$ & $<0.5,0.5,0.45>$ & $<0.738,0.263,0.210>$ \\
\hline \multirow{6}{*}{$A_{3}$} & $a_{31}$ & $<0.205,0.756,0.783>$ & $<0.627,0.373,0.319>$ & $<0.534,0.466,0.416>$ & $<0.081,0.869,0.919>$ \\
\hline & $a_{32}$ & $<0.761,0.239,0.187>$ & $<0.623,0.377,0.323>$ & $<0.534,0.466,0.416$ & $<0.133,0.817,0.867>$ \\
\hline & $a_{33}$ & $<0.247,0.720,0.736>$ & $<0.707,0.293,0.240>$ & $<0.554,0.446,0.395>$ & $<1,0,0>$ \\
\hline & $a_{34}$ & $<0.836,0.164,0.109>$ & $<0.589,0.410,0.354>$ & $<0.534,0.466,0.416>$ & $<0.1,0.85,0.9>$ \\
\hline & $a_{35}$ & $<0.474,0.526,0.476>$ & $<0.579,0.421,0.370>$ & $<0.65,0.35,0.3>$ & $<0.860,0.140,0.085>$ \\
\hline & $a_{36}$ & $<0.576,0.424,0.364>$ & $<0.612,0.388,0.337>$ & $<0.474,0.526,0.476>$ & $<0.2,0.75,0.8>$ \\
\hline \multirow{3}{*}{$A_{4}$} & $a_{41}$ & $<0.825,0.175,0.121>$ & $<0.533,0.466,0.416>$ & $<0.777,0.223,0.172>$ & $<0.701,0.299,0.244>$ \\
\hline & $a_{42}$ & $<0.857,0.143,0.088>$ & $<0.5,0.5,0.45>$ & $<0.8,0.2,0.15>$ & $<0.608,0.392,0.342>$ \\
\hline & $a_{43}$ & $<0.886,0.114,0.062>$ & $<0.530,0.469,0.418>$ & $<0.8,0.2,0.15>$ & $<0.625,0.375,0.325>$ \\
\hline
\end{tabular}

TABle 8: Pairwise comparison vector of the most important criterion.

\begin{tabular}{lcccc}
\hline Criteria & $A_{1}$ & $A_{2}$ & $A_{3}$ & $A_{4}$ \\
\hline Best criterion: $A_{1}$ & 1 & 6 & 2 & 4 \\
\hline
\end{tabular}

TABLE 9: Pairwise comparison vector of the least important criterion.

\begin{tabular}{lcccc}
\hline Criteria & $A_{1}$ & $A_{2}$ & $A_{3}$ & $A_{4}$ \\
\hline Worst criterion: $A_{2}$ & 6 & 1 & 4 & 2 \\
\hline
\end{tabular}

the preference values of the best criteria $\left(A_{1}\right)$ over criterion $\left(A_{2}\right)$, criterion $\left(A_{3}\right)$, and criterion $\left(A_{4}\right)$ are 6,2 , and 4 , respectively. The preference values of criteria $\left(A_{1}\right)$, criterion $\left(A_{3}\right)$, and criterion $\left(A_{4}\right)$ over the worst criterion $\left(A_{2}\right)$ are 6,4 , and 2, respectively, as shown in Table 9. Finally, the weight vector of criteria $w^{*}=\left\{w_{1}^{*}, w_{2}^{*}, w_{3}^{*}, w_{4}^{*}\right\}$ is calculated by Equation (11), and we can get $w_{1}^{*}=0.5098, w_{2}^{*}=0.0784$, $w_{3}^{*}=0.2745, w_{4}^{*}=0.1373$, and $\xi^{*}=0.0392$. For the consistency ratio based on the BWM method, as $a_{B W}=a_{12}=6$, the consistency index for this problem is 3.00 (shown in Table 4), and the consistency ratio according to equation (9) is $0.0392 /$ $3.00 \approx 0.0131$, which illustrates a very good consistency.

Afterwards, the weights of indices can be computed by applying the entropy weight method outlined in Section 4.3.2. First, by normalizing the evaluation indices by utilizing equation (12), we shall get the evaluation matrix $R=\left(\beta_{i j}\right)$, which is shown in Table 10 . Then, we can obtain the entropy weight of each index based on the normalized evaluation matrix.

At last, index weights can be acquired by combining the subjective weights of criteria with the entropy weights of indices, which is obtained as in Table 11.
5.3. The Sequence of Restoration Methods. We use the TODIM method based on MCGDM introduced in Section 4.4 to solve the restoration methods selection problem. First, we calculate the final dominance of restoration methods $X_{i}$ over each other plan $X_{k}$ under each index $a_{i j}$ by using equation (16), and the parameter $\theta$ is usually 1 ; the result can be shown in Table 12. Then, the global value of each restoration method is obtained by equation (17). Finally, according to value $\varepsilon_{i}$, we could sort out restoration methods selection $X_{i}$, which is obtained as in Table 13. Hence, the most appropriate restoration method for $C_{1}$ is " $X_{1}$," namely, stabilizing by outer iron hoop.

5.4. Sensitivity Analysis. The parameter $\theta$ in TODIM is the loss attenuation coefficient that controls the influence caused when a loss occurs. We believe that if $\theta<1$, the loss is amplified; if $\theta>1$, the loss is attenuated. The prospect theory states that, in general, individuals are more sensitive to losses than gains, which suggests that $\theta<1$. Therefore, the value of this parameter greatly affects the ranking order of the alternatives. Choosing a small $\theta$ means we are looking for an alternative which provides less loss under all conditions; on the contrary, if we choose a big $\theta$, then we are looking for an alternative which provide greater benefits, even if we have losses on certain criteria [46]. For the purpose of analyzing the influence of different values of $\theta$ on the final ranking orders, we take different $\theta$ values to calculate the examples for restoration methods to check the stability of the ranking results. The results are outlined in Table 14 and Figure 3. We can see the different ranking orders calculated by different $\theta$ clearly. When $\theta \geq 0.8$, the ranking order is the same as in the previous example, and the ranking order is $X_{1}>X_{3}>X_{2}>X_{4}$; when the loss attenuation coefficient becomes smaller, $\theta \leq 0.5, X_{3}$ and $X_{1}$ are replaced, and the ranking result becomes $X_{3}>X_{1}>X_{2}>X_{4}$. This proves that when the loss is 
TABLE 10: The normalized evaluation matrix.

\begin{tabular}{|c|c|c|c|c|c|}
\hline \multirow{2}{*}{ Criteria } & \multirow{2}{*}{ Index } & \multicolumn{4}{|c|}{ Alternatives } \\
\hline & & $X_{1}$ & $X_{2}$ & $X_{3}$ & $X_{4}$ \\
\hline \multirow{6}{*}{$A_{1}$} & $a_{11}$ & $<0.719,0.281,0.229>$ & $<0.5,0.5,0.45>$ & $<0.777,0.223,0.172>$ & $<0.465,0.525,0.491>$ \\
\hline & $a_{12}$ & $<0.761,0.239,0.187>$ & $<0.506,0.494,0.442>$ & $<0.8,0.2,0.15>$ & $<0.545,0.455,0.398>$ \\
\hline & $a_{13}$ & $<0.704,0.296,0.240>$ & $<0.648,0.352,0.298>$ & $<0.733,0.267,0.214>$ & $<0.262,0.709,0.715>$ \\
\hline & $a_{14}$ & $<0.625,0.375,0.325>$ & $<0.473,0.526,0.476>$ & $<0.777,0.223,0.172>$ & $<0.453,0.547,0.496>$ \\
\hline & $a_{15}$ & $<0.625,0.375,0.325>$ & $<0.579,0.421,0.370>$ & $<0.8,0.2,0.15>$ & $<0.159,0.791,0.841>$ \\
\hline & $a_{16}$ & $<0.809,0.224,0.210>$ & $<0.508,0.493,0.544>$ & $<0.814,0.218,0.204>$ & $<0,1,1>$ \\
\hline \multirow{4}{*}{$A_{2}$} & $a_{21}$ & $<0.719,0.303,0.310>$ & $<0.235,0.765,0.817>$ & $<0.466,0.534,0.584>$ & $<0.286,0.714,0.768>$ \\
\hline & $a_{22}$ & $<0.819,0.231,0.181>$ & $<0.435,0.565,0.616>$ & $<0.587,0.413,0.464>$ & $<0.235,0.765,0.817>$ \\
\hline & $a_{23}$ & $<0.65,0.35,0.4>$ & $<0.175,0.825,0.879>$ & $<0.544,0.456,0.507>$ & $<0.234,0.766,0.831>$ \\
\hline & $a_{24}$ & $<0.695,0.320,0.342>$ & $<0.2,0.8,0.85>$ & $<0.5,0.5,0.55>$ & $<0.262,0.738,0.790\rangle$ \\
\hline \multirow{6}{*}{$A_{3}$} & $a_{31}$ & $<0.205,0.756,0.783>$ & $<0.627,0.373,0.319>$ & $<0.534,0.466,0.416>$ & $<0.081,0.869,0.919>$ \\
\hline & $a_{32}$ & $<0.761,0.239,0.187>$ & $<0.623,0.377,0.323>$ & $<0.534,0.466,0.416$ & $<0.133,0.817,0.867>$ \\
\hline & $a_{33}$ & $<0.753,0.280,0.264>$ & $<0.293,0.707,0.760>$ & $<0.446,0.554,0.605>$ & $<0,1,1>$ \\
\hline & $a_{34}$ & $<0.836,0.164,0.109>$ & $<0.589,0.410,0.354>$ & $<0.534,0.466,0.416>$ & $<0.1,0.85,0.9>$ \\
\hline & $a_{35}$ & $<0.474,0.526,0.476>$ & $<0.579,0.421,0.370>$ & $<0.65,0.35,0.3>$ & $<0.860,0.140,0.085>$ \\
\hline & $a_{36}$ & $<0.424,0.576,0.636>$ & $<0.388,0.612,0.663>$ & $<0.526,0.474,0.524>$ & $<0.8,0.25,0.2>$ \\
\hline \multirow{3}{*}{$A_{4}$} & $a_{41}$ & $<0.825,0.175,0.121>$ & $<0.533,0.466,0.416>$ & $<0.777,0.223,0.172>$ & $<0.701,0.299,0.244>$ \\
\hline & $a_{42}$ & $<0.857,0.143,0.088>$ & $<0.5,0.5,0.45>$ & $<0.8,0.2,0.15>$ & $<0.608,0.392,0.342>$ \\
\hline & $a_{43}$ & $<0.886,0.114,0.062>$ & $<0.530,0.469,0.418>$ & $<0.8,0.2,0.15>$ & $<0.625,0.375,0.325>$ \\
\hline
\end{tabular}

TABLE 11: The weights of criteria and index for restoration methods selection.

\begin{tabular}{lccc}
\hline Criteria & Weight & Index & Weight \\
\hline & & $a_{11}$ & 0.0705 \\
& & $a_{12}$ & 0.0882 \\
$A_{1}$ & 0.5098 & $a_{13}$ & 0.1025 \\
& & $a_{14}$ & 0.0570 \\
& & $a_{15}$ & 0.1050 \\
& & $a_{16}$ & 0.1232 \\
\hline \multirow{3}{*}{$A_{2}$} & & $a_{21}$ & 0.0142 \\
& 0.0784 & $a_{22}$ & 0.0132 \\
& & $a_{23}$ & 0.0135 \\
& & $a_{24}$ & 0.0140 \\
\hline \multirow{3}{*}{$A_{3}$} & & $a_{31}$ & 0.0539 \\
& & $a_{32}$ & 0.0532 \\
& 0.2745 & $a_{33}$ & 0.0640 \\
& & $a_{34}$ & 0.0578 \\
$A_{4}$ & & $a_{35}$ & 0.0444 \\
& & $a_{36}$ & 0.0368 \\
\hline
\end{tabular}

TABLE12: The final dominance of restoration methods alternatives $(\theta=1)$.

\begin{tabular}{ccccc}
\hline & $X_{1}$ & $X_{2}$ & $X_{3}$ & $X_{4}$ \\
\hline$X_{1}$ & 0 & -1.5129 & -0.0042 & -2.1098 \\
$X_{2}$ & 1.5129 & 0 & 1.4057 & -0.9879 \\
$X_{3}$ & 0.0042 & -1.4057 & 0 & -2.0915 \\
$X_{4}$ & 2.0081 & 0.8676 & 2.0915 & 0 \\
\hline
\end{tabular}

magnified, the decision-makers tend to choose $X_{3}$. When the loss is reduced, that is, the larger the loss attenuation coefficient, the lower the degree of evasion by the decision-
TABLE 13: Ranking order of restoration methods by $\varepsilon_{i}$.

\begin{tabular}{lcc}
\hline Alternatives & Values $\varepsilon_{i}$ & Ranking orders \\
\hline$X_{1}$ & 1 & 1 \\
$X_{2}$ & 0.3601 & 3 \\
$X_{3}$ & 0.9963 & 2 \\
$X_{4}$ & 0 & 4 \\
\hline
\end{tabular}

TABle 14: Ranking order of restoration methods with different $\theta$.

\begin{tabular}{lccccc}
\hline $\begin{array}{l}\text { Different values of } \\
\theta\end{array}$ & \multicolumn{5}{c}{ Values $\varepsilon_{i}$} \\
& $X_{1}$ & $X_{2}$ & $X_{3}$ & $X_{4}$ & Ranking orders \\
\hline$\theta=2.25$ & 1 & 0.2803 & 0.9464 & 0 & $X_{1}>X_{3}>X_{2}>X_{4}$ \\
$\theta=2.0$ & 1 & 0.3246 & 0.9893 & 0 & $X_{1}>X_{3}>X_{2}>X_{4}$ \\
$\theta=1.5$ & 1 & 0.3386 & 0.9922 & 0 & $X_{1}>X_{3}>X_{2}>X_{4}$ \\
$\theta=1.0$ & 1 & 0.3601 & 0.9963 & 0 & $X_{1}>X_{3}>X_{2}>X_{4}$ \\
$\theta=0.8$ & 1 & 0.3716 & 0.9985 & 0 & $X_{1}>X_{3}>X_{2}>X_{4}$ \\
$\theta=0.5$ & 0.9973 & 0.3925 & 1 & 0 & $X_{3}>X_{1}>X_{2}>X_{4}$ \\
$\theta=0.3$ & 0.9937 & 0.4101 & 1 & 0 & $X_{3}>X_{1}>X_{2}>X_{4}$ \\
\hline
\end{tabular}

maker, the decision-makers tend to choose $X_{1}$. It means that adjusting the decision-maker's risk aversion tendency will affect the ranking results, and it also indicates that the psychological behavior of the decision-maker will affect one's decision-making. The results of sensitivity analysis show that the proposed method can well express the cognition of decision-makers, quantify the uncertainty to a certain extent, and avoid the loss and distortion of information as much as possible. Therefore, to an extent, the feasibility and robustness of the proposed model is verified.

5.5. Comparison Analysis. As explained in Section 2, the model is proposed to select restoration methods for wood components of Chinese architectures. In order to verify that 


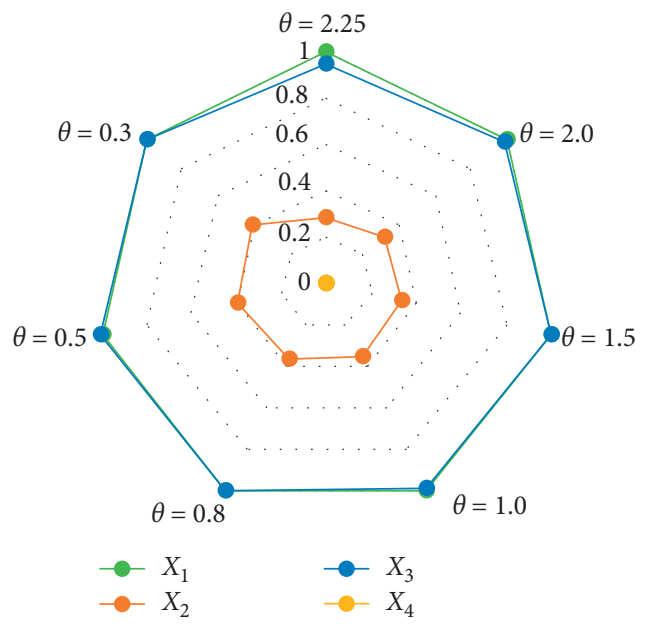

FIGURE 3: The radar chart displaying the result of sensitivity analysis.

TABLE 15: The comparison of different ranking methods.

\begin{tabular}{lccccrr}
\hline \multirow{2}{*}{ Alternatives } & \multicolumn{2}{c}{ TOPSIS } & \multicolumn{3}{c}{ VIKOR } \\
& Close degree & Ranking orders & $S_{i}$ & $R_{i}$ & $Q_{i}$ & Ranking orders \\
\hline$X_{1}$ & 0.6666 & & 0.3119 & 0.0531 & 0.0099 \\
$X_{2}$ & 0.4413 & $X_{3}>X_{1}>X_{2}>X_{4}$ & 0.5142 & 0.0843 & 0.4615 & $X_{1}>X_{3}>X_{2}>X_{4}$ \\
$X_{3}$ & 0.7533 & & 0.3276 & 0.0508 & 0.0244 & \\
$X_{4}$ & 0.3655 & & 0.6328 & 0.0244 & 1.0000 & \\
\hline
\end{tabular}

the proposed model can be validly and practically used to identify the most suitable restoration method for specific wood components, we employ the TOPSIS [47] and VIKOR [48] to make comparative analysis of the above empirical study. The ranking order of the four restoration methods obtained by TOPSIS and VIKOR is outlined in Table 15. From Table 15, the ranking result of the proposed model is consistent with those computed by the TOPSIS when $\theta \leq 0.5$. And the ranking order of the case study is the same as those computed by VIKOR. But in the calculation of the VIKOR method, $Q\left(X_{1}\right)-Q\left(X_{3}\right)<0.25$, it means the ranking results of $X_{1}$ and $X_{3}$ are similar, so both $X_{1}$ and $X_{3}$ are close to the ideal restoration method. It can be seen that TODIM can better reflect the psychological behavior of the decisionmakers and can flexibly respond to the trend of risk aversion. Therefore, the validity of the proposed model can be verified. Compared with the existing approaches for restoration methods selection for wood components of Chinese architectures, the advantages of the model proposed can be summarized as follows:

(i) The model comprehensively considers the subjective and objective criteria in the index system. According to the index system, the evaluation information characterized by multigranularity linguistic terms is obtained from the experts, and the single-valued neutrosophic set is used to quantify the expert evaluation information. Therefore, this makes the selection of restoration methods more realistic. (ii) In order to solve the ranking problem with unknown criteria weights and index weights in the TODIM scheme, combine BWM method and entropy weight method to calculate the criteria and index weights, respectively. As a result, index weights are reliable and more accurate.

(iii) The proposed model employs TODIM prioritized alternative restoration methods. The TODIM method fully considers the risk aversion of decisionmakers and reflects the preferences of decisionmakers by adjusting parameter $\theta$. Hence, the proposed restoration methods selection model based on TODIM with single-valued neutrosophic sets is a suitable method to solve multicriteria group decision-making problems considering the psychological behavior of decision-makers.

\section{Conclusion}

A selection model of restoration methods for wood components of Chinese ancient architectures has been developed in this paper, which is helpful to assisting experts to solve the problem of selecting appropriate restoration methods. Subjective and objective criteria have been simultaneously utilized in the establishment of index system. Moreover, single-valued neutrosophic sets are employed to characterize the evaluation information given by experts in multigranularity language terms and to quantify the assessment information. In addition, the BWM method and entropy 
weight method are used to obtain the criteria and index weights. Finally, the TODIM method has been used to get the prioritization of restoration methods. The proposed model has been conducted to an empirical study in Fuhoutang for restoration methods selection of a specific wood component. The detailed process of the model has been illustrated in this paper. Furthermore, the sensitivity analysis and comparison analysis proved the reliability and effectiveness of the proposed selection model.

In conclusion, the proposed model not only improves extant approaches in the field of restoration methods decision-making for Chinese ancient architectures but also gives rational support to experts in the process of decisionmaking. So, both theoretical development and practical application are reflected in this work. There are several implications for possible directions of further study. First, more types of information of restoration methods selection should be taken into consideration in the proposed model in future studies. Second, the model can be extended to apply other decision methods, such as TOPSIS and VIKOR. Finally, the proposed selection model can also be applied to other kinds of components of Chinese ancient architectures besides wood components.

\section{Data Availability}

The data used to support the findings of this study are included within the article.

\section{Conflicts of Interest}

The authors declare that there are no conflicts of interest.

\section{Acknowledgments}

The authors would like to thank the experts who participated in the survey. This work was supported by the Human Philosophy Social Science Fund Projects (no. 18YBA150), the Later Support from National Social Science Fund (no. 19FGLB011), the Key Project of Hunan Education Department (no. 18A201), the Think Tank Project of Hunan Social Sciences Federation (no. ZK2019006), and the Hunan Provincial Natural Science Fund (nos. 2019JJ40088 and 2019JJ40479).

\section{References}

[1] M. Yu, Y. Oda, D. Fang, and J. Zhao, "Advances in structural mechanics of Chinese ancient architectures," Frontiers of Architecture and Civil Engineering in China, vol. 2, no. 1, pp. 1-25, 2008.

[2] A. Zaboklicki and M. Gebski, "Continuity of wooden beams as a method of reinforcement and preservation of timber structures at monumental buildings," Transaction on the Built Environment, vol. 26, pp. 541-546, 1997.

[3] N. Orlando, Y. Taddia, E. Benvenuti, B. Pizzo, and C. Alessandri, "End-repair of timber beams with laterallyloaded glued-in rods: experimental trials and failure prediction through modelling," Construction and Building Materials, vol. 195, pp. 623-637, 2019.
[4] T. Koike, "Progress in development of epoxy resin systems based on wood biomass in Japan," Polymer Engineering \& Science, vol. 52, no. 4, pp. 701-717, 2012.

[5] N. Dourado, F. A. M. Pereira, M. F. S. F. de Moura, and J. J. L. Morais, "Repairing wood beams under bending using carbon-epoxy composites," Engineering Structures, vol. 34, pp. 342-350, 2012.

[6] M. V. Casals, V. R. Rodríguez, and C. L. Badia, “Timber structure repair of an emblematic catalan industrial building with wood grafts and epoxy resins," Advanced Materials Research, vol. 778, pp. 998-1005, 2013.

[7] M. Khelifa and A. Celzard, "Numerical analysis of flexural strengthening of timber beams reinforced with CFRP strips," Composite Structures, vol. 111, pp. 393-400, 2014.

[8] A. Baratta and I. Corbi, "FRP composites retrofitting for protection of monumental and ancient constructions," The Open Construction and Building Technology Journal, vol. 6, no. 1, pp. 361-367, 2012.

[9] A. M. Forster and B. Kayan, "Maintenance for historic buildings: a current perspective," Structural Survey, vol. 27, no. 3, pp. 210-229, 2009.

[10] L. Fregonese, G. Barbieri, L. Biolzi, M. Bocciarelli, A. Frigeri, and L. Taffurelli, "Surveying and monitoring for vulnerability assessment of an ancient building," Sensors, vol. 13, no. 8, pp. 9747-9773, 2013.

[11] Z. Gao, D. Ma, W. Wang, X. Guo, and Q. Ge, "Development and application of ancient timber buildings structural condition assessment model based on a fuzzy matter-element model that includes asymmetric proximity," Mathematical Problems in Engineering, vol. 2018, Article ID 7426915, 12 pages, 2018.

[12] P. B. Lourenço, "Recommendations for restoration of ancient buildings and the survival of a masonry chimney," Construction and Building Materials, vol. 20, no. 4, pp. 239-251, 2006.

[13] R. Wang, X. Zhang, and T. T. Han, "Economic comparison for the protection scheme of ancient building based on BIM," Applied Mechanics and Materials, vol. 496-500, pp. 25532556, 2014.

[14] F. Smarandache, "A Unifying Field in Logics: Neutrosophic Logic. Neutrosophy," Neutrosophic Set, Neutrosophic Probability, American Research Press, Rehoboth, NM, USA, 1999.

[15] S.-Z. Luo, P.-F. Cheng, J.-Q. Wang, and Y.-J. Huang, "Selecting project delivery systems based on simplified neutrosophic linguistic preference relations," Symmetry, vol. 9, no. 8, p. 151, 2017.

[16] P. Liu, P. Wang, and J. Liu, "Normal neutrosophic frank aggregation operators and their application in multi-attribute group decision making," International Journal of Machine Learning and Cybernetics, vol. 10, no. 5, pp. 833-852, 2019.

[17] H.-Y. Zhang, P. Ji, J.-Q. Wang, and X.-H Chen, "A novel decision support model for satisfactory restaurants utilizing social information: a case study of tripadvisor.com," Tourism Management, vol. 59, pp. 281-297, 2017.

[18] D.-P. Li, J.-Q. He, P.-F. Cheng, J.-Q. Wang, and H.-Y. Zhang, "A novel selection model of surgical treatments for early gastric cancer patients based on heterogeneous multicriteria group decision-making," Symmetry, vol. 10, no. 6, pp. 223252, 2018.

[19] Z.-P. Tian, R.-X. Nie, and J.-Q. Wang, "Probabilistic linguistic multi-criteria decision-making based on evidential reasoning and combined ranking methods considering decision-makers' psychological preferences," Journal of the Operational Research Society, vol. 71, no. 5, pp. 700-717, 2019. 
[20] P. Liu, S. Cheng, and Y. Zhang, "An extended multi-criteria group decision-making PROMETHEE method based on probability multi-valued neutrosophic sets," International Journal of Fuzzy Systems, vol. 21, no. 2, pp. 388-406, 2019.

[21] J. Rezaei, "Best-worst multi-criteria decision-making method," Omega, vol. 53, pp. 49-57, 2015.

[22] X. S. You, T. Chen, and Q. Yang, "Approach to multi-criteria group decision-making problems based on the best-worstmethod and ELECTRE method," Symmetry, vol. 8, no. 9, pp. 1-16, 2016.

[23] R. Lourenzutti and R. A. Krohling, "A study of TODIM in a intuitionistic fuzzy and random environment," Expert Systems with Applications, vol. 40, no. 16, pp. 6459-6468, 2013.

[24] D. Kahneman and A. Tversky, "Prospect theory: an analysis of decision under risk," Econometrica, vol. 47, no. 2, pp. 263292, 1979.

[25] H.-G. Peng, J.-Q. Wang, and H.-Y. Zhang, "Multi-criteria outranking method based on probability distribution with probabilistic linguistic information," Computers \& Industrial Engineering, vol. 141, Article ID 106318, 2020.

[26] R.-X Nie and J.-Q. Wang, "Prospect theory-based consistency recovery strategies with multiplicative probabilistic linguistic preference relations in managing group decision making," Arabian Journal for Science and Engineering, vol. 45, no. 3, pp. 2113-2130, 2020.

[27] P. Liu and F. Teng, "Probabilistic linguistic TODIM method for selecting products through online product reviews," Information Sciences, vol. 485, pp. 441-455, 2019.

[28] P. Ren, Z. Xu, and X. Gou, "Pythagorean fuzzy TODIM approach to multi-criteria decision making," Applied Soft Computing, vol. 42, pp. 246-259, 2015.

[29] P. Liu, L. Rong, and F. Teng, "The evaluation of ecosystem health based on hybrid TODIM method for Chinese case," Technological and Economic Development of Economy, vol. 25, no. 3, pp. 542-570, 2019.

[30] G. Zhang, J. Q. Wang, and T. L. Wang, "Multi-criteria group decision-making method based on TODIM with probabilistic interval-valued hesitant fuzzy information," Expert Systems, vol. 36, no. 4, 2019.

[31] L. Xie, J. He, P. Cheng, R. Xiao, and X. Zhou, "A multi-criteria 2-tuple linguistic group decision-making method based on TODIM for cholecystitis treatments selection," IEEE Access, vol. 7, pp. 127967-127986, 2019.

[32] P. Liu and X. You, "Improved TODIM method based on linguistic neutrosophic numbers for multicriteria group decision-making," International Journal of Computational Intelligence Systems, vol. 12, no. 2, pp. 544-556, 2019.

[33] A. Kharal, "A neutrosophic multi-criteria decision making method," New Mathematics and Natural Computation, vol. 10, no. 2, pp. 143-162, 2014.

[34] H. B. Wang, Y. Q. Zhang, R. Sunderraman, and F. Smarandache, "Single valued neutrosophic sets," Fuzzy Sets, Rough Sets and Multivalued Operations and Applications, vol. 3, pp. 33-39, 2011.

[35] J.-J. Peng, J.-Q. Wang, H.-Y. Zhang, and X.-H. Chen, “An outranking approach for multi-criteria decision-making problems with simplified neutrosophic sets," Applied Soft Computing, vol. 25, pp. 336-346, 2014.

[36] P. Majumdar and S. K. Samanta, "On similarity and entropy of neutrosophic sets," Journal of Intelligent \& Fuzzy Systems, vol. 26, no. 3, pp. 1245-1252, 2014.

[37] R. P. Tan, W. D. Zhang, and L. L. Chen, "Study on emergency group decision making method based on VIKOR with single valued neutrosophic sets," Journal of Safety Science and Technology, vol. 13, no. 2, pp. 79-84, 2017.

[38] P. Biswas, S. Pramanik, and B. C. Giri, "TOPSIS method for multi-attribute group decision-making under single-valued neutrosophic environment," Neural Computing and Applications, vol. 27, no. 3, pp. 727-737, 2015.

[39] J. Ye, "A multicriteria decision-making method using aggregation operators for simplified neutrosophic sets," Journal of Intelligent \& Fuzzy Systems, vol. 26, no. 5, pp. 2459-2466, 2014.

[40] X.-Y. Zhang, H.-Y. Zhang, and J.-Q. Wang, "Discussing incomplete 2-tuple fuzzy linguistic preference relations in multi-granular linguistic MCGDM with unknown weight information," Soft Computing, vol. 23, no. 6, pp. 2015-2032, 2017.

[41] C. Song, X.-K. Wang, P.-F Cheng, J.-Q. Wang, and L. Li, "SACPC: a framework based on probabilistic linguistic terms for short text sentiment analysis," Knowledge-Based Systems, 2020.

[42] J. Rezaei, "Best-worst multi-criteria decision-making method: some properties and a linear model," Omega, vol. 64, pp. 126-130, 2016.

[43] H. A. Mahdiraji, S. Arzaghi, G. Stauskis, and E. K. Zavadskas, "A hybrid fuzzy BWM-COPRAS method for analyzing key factors of sustainable architecture," Sustainability, vol. 10, no. 5, p. 1626, 2018.

[44] F.-c. Yu, G.-h. Fang, and R. Shen, "Study on comprehensive early warning of drinking water sources for the Gucheng lake in China," Environmental Earth Sciences, vol. 72, no. 9, pp. 3401-3408, 2014.

[45] L. Gomes and M. Lima, "TODIM: basics and application on multicriteria ranking of projects with environmental impacts," Foundations of Computing and Decision Sciences, vol. 16, pp. 113-127, 1992.

[46] R. Lourenzutti and R. A. Krohling, "TODIM based method to process heterogeneous information," Procedia Computer Science, vol. 55, pp. 318-327, 2015.

[47] D. H. Liu, Y. Y. Liu, and L. Z. Wang, "Distance measure for fermatean fuzzy linguistic term sets based on linguistic scale function: an illustration of the TODIM and TOPSIS methods," International Journal of Intelligent Systems, vol. 34, no. 2, pp. 2807-2834, 2019.

[48] L. Lei and W. F. Zhang, "Extended VIKOR method for multilevel hybrid multi-attribute group decision making," in Proceedings of the 25th Chinese Control and Decision Conference (CCDC), pp. 1718-1722, Guiyang, China, May 2013. 